\title{
Aegäische, besonders kretische Namen bei den Etruskern.
}

\section{Von A. Kannengiesser.}

Für die Herkunft der Etrusker aus dem kretisch-karischen oder, wie ich nach Ficks Vorgange ihn lieber nennen will, hattidischen Völkerkreise mehren sich von Tag zu Tage die Beweise, besonders archäologische und mythologische. Ein genügender, linguistischer Beweis steht aber immer noch aus und kann m. E. nach dem heutigen Stande unserer Kenntnis der etruskischen und der hattidischen Sprachen vorerst auch nur an der Hand der uns vorliegenden einerseits etruskischen bezw. etruskisch-lateinischen und andererseits hattidischen Figennamen erbracht werden. Einen Beitrag hierzu soll die vorliegende Arbeit liefern. Als Gebiete, welche von vorgriechischer Bevölkerung bewohnt waren, kommen als Heimat der Etrusker die Länder am ägäischen Meere und unter diesen in erster Linie die Insel Kreta in Betracht. Fick ${ }^{1}$ ) hat das Namenmaterial, das in kretischen Ortsnamen vorliegt, eingehend untersucht und das Griechische vom Vorgriechischen scharf gesondert; die Ergebnisse, zu denen er gelangt ist, sind im allgemeinen anerkannt worden. Sehr zweifellaft aber ist es, ob die Kydonen, wie Fick meint, thrakisch-phrygischen Ursprungs sind oder auch zu der urkretischen Bevölkerung gehören, ferner, ob eine strenge Unterscheidung: von pelasgischer, lelegischer und urkretischer Bevölkerung sich im einzelnen durchführen lässt oder ob wir uns in der Regel damit begnügen müssen, nur im allgemeinen von den Völkern, die am ägäischen Meer wohnten, als hattidischen d. h. weder indogermanischen noch semitischen Völkern zu sprechen.

Für unseren Zweck genügt jedenfalls das letztere. Auch die Frage, ob ein Teil der alten Bevölkerung Kretas thrakisch-phrygisch war oder nicht, will ich vorläufig auf sich beruhen lassen und mich damit begnügen zu beweisen, dass das nicht griechiseheoder vorgriechische Namenmaterial Kretas zu einem sehr grossen Teile in Etrurien wiederkehrt ${ }^{2}$ ).

Ehe wir in die Untersuchung eintreten, bedarf es einiger Worte über

1) Die griechischen Ortsnamen als Quelle der Vorgeschichte Griechenlands, Göt. tingen 1905, und Hattiden und Danubier, Göttingen 1909.

2) Dass der Name Kvdwvio nicht thrakisch ist, soll unten S. 38 gezeigt werden. 
die Grundsätze, welche für die Vergleichung der auf beiden Gebieten vorkommenden Namen, soweit sie für die Frage nach der Herkunft der Etrusker in Betracht kommen, massgebend sein müssen. Selbstverständlich müssen die etruskischen Namen wirklich etruskisches Sprachgut und nicht etwa mit etruskischen Suffixen versehene Namen von Italikern oder illyrischvenetischen Völkern sein. $O b$ ein Name etruskischer Herkunft ist, lässt sich häufig schwer oder gar nicht bestimmen; es bleiben daber besser alle Namen fort, deren etruskische Herkunft nicht wenigstens im höchsten Grade wahrscheinlich ist. Als Merkmale für einen solchen Ursprung gelten: erstens das Vorkommen eines Namens in einer alten Inschrift etruskischer Sprache, zweitens häufiges Vorkommen des Namens auf etruskischem Gebiet, drittens die Zugehörigkeit desselben zu einer grösseren spezifisch etruskischen Namengruppe, viertens das sonstige Fehlen des Namensstammes bei den nichtetruskischen Völkern Italiens, wenn die Namensform etruskisch ist. Ferner müssen die zum Vergleich herangezogenen Namen beider Gebiete alt sein; insbesondere dürfen sie nicht erst von Inschriften aus der Zeit des römischen Weltreichs stammen, in welcher Leute aus allen Gegenden in Italien zusammenströmten und Römer draussen als Beamte oder Kaufleute wohnten ${ }^{1}$. Es ist nicht meine Aufgabe, alle Namen, die möglicherweise denselben Ursprung haben können, zusammenzustellen, sondern nur das soll hier herangezogen werden, was für die Herkunft etruskischer Namen wirklich beweisend ist.

In dem Gange meiner Untersuchung folge ich im allgemeinen Ficks Schrift über die Vorgriechischen Ortsnamen, die ich mit $F$. zitiere, und füge dann ein paar von diesem nicht behandelte Ortsnamen sowie einige kretische Personennamen hinzu. Das Material für die etruskischen und sonstigen italischen Namen entnehme ieh in der Regel, auch wenn dies nicht besonders bemerkt ist, W. Schulzes Werk Zur Geschichte lateinischer Eigennamen, das ich mit Sch. zitiere.

Ueber den Namen der Kydonen handle ich unten bei $K v \dot{\tau} \tau$, ich beginne mit $F$. S. 17 :

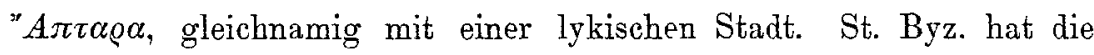

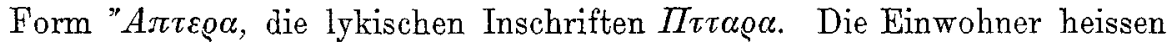

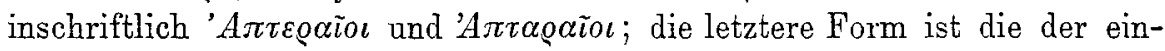
heimischen Insehrift Smlg. Nr. 4942.

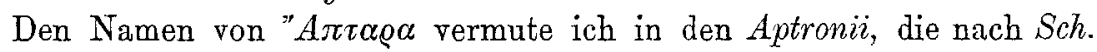
S. 111 , auf den alten Grabinschriften Praenestes, CIL XIV 3063 ff. auftauchen, um dann ganz aus der Ueberlieferung zu verschwinden ". Praeneste ist die Fundgrube von zahlreichen etruskischen Namen, Schulze meint geradezu, es mit etr. presnte verbinden zu können ${ }^{2}$ ).

1) Ausnahmsweise wird indes auch ein solcher Name berïcksichtigt werden können.

2) Fick vergleicht zur Endung zwei andere lykische Städte, nämlich Mór $\alpha \varrho \propto$ und

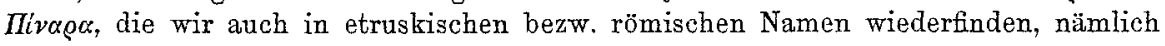


F. S. 18: Mvंxvos. Den Namen dieses bei Kydonia fliessenden Ba-

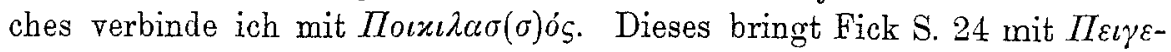
$\lambda \alpha \sigma o ́ s$ in Karien und $\Pi v \dot{\gamma} \varepsilon \lambda \alpha$ in Lydien in Verbindung. Ir $\gamma \varepsilon \lambda \alpha \alpha$ stimmt jedenfalls zu Puculeius, Buculeius, Bucleius, etr. puctis und in letzter linie zu etr. puce (CIE 1639, 2609 Clusium). Hierzu gehört das Gentilicium pucna (CIE 2610, ebenfalls Clusium), dessen Name in Kreta in dem Flussnamen $\Pi v \dot{x} x$ os wiederkehrt, der demnach der Bach des Geschlechtes der pucna sein wird, wie der Sarnus des Geschlechtes der Sarii (cf. Sch. S. 571).

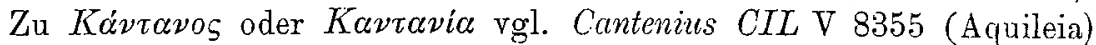
8651 (Iulium Carnicum), cantini CIE 4204 (Perusia), can $\imath u s a 902,1257$ (Clusium) etc., m. O. Cantiniano in Toseana etc. ${ }^{1}$. Oestlich von Kantanos lag "Eגv@os. Vermutlich ist am Anfang ein $f$ ausgefallen, sodass der Name zu dem etr. Vornamen velu gehört, dieser ist wiederum eine Erweiterung des Vornamens rel, der im Etr. ebenso häufig ist wie Gainus im Lateinischen. Zu "Eגv@os ist speziell auf Velurizus (Pompeii) und etr. velrnal CIE 3402 zu verweisen ${ }^{2}$ ).

Der Hafen von Elyros hiess $\Sigma v i \alpha$, wir haben den dazu gehörigen Gentilnamen in etr. suie, lat. Soius und Suizus, dazu gehören ferner svea, Soenius, Sueius etc. (Sch. 233). Die von Fick herangezogenen Namen, die mit demselben Stamm gebildet sind, finden wir sämtlich genan in Italien wieder:

$\Sigma v ́ \varepsilon \sigma \sigma c$ (lykisch) in Suessa 1. in Latium, 2. im Aurunkerland; dazu

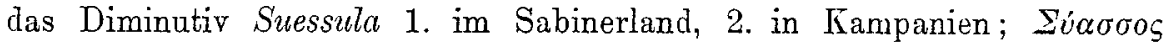
in Phrygien $=$ Suasa in der gallischen Mark. $\mathrm{Zu}$ derselben Gruppe ge-

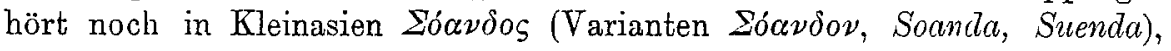
welches mit seiner kleinasiatischen Diminutivendung ${ }^{3}$ ) genau dem italischen Suessulla entspricht.

Tá@@a, welches nach St. Byz. auch im Pontus und in Lydien vorkommt, gehört zu tarna, Tarius, Taronins, Tarronius, Tarutius etc. (Sch. S. 96 f.), und wahrscheinlich bängen hiermit auch Tarraco und Tarracina

in der weit verbreiteten Sippe der patruni oder petruni, sowie in dem, wiederum auch pränestinischen, Patronius (CIL XIV, 2878), Mivcec ist u. a. in der Familie der Pinarii vertreten, auf deren asiatischen Ursprung schon Hommel hingewiesen hat (Grundriss S. 65).

1) Fick vergleicht zur Endung " $A v \delta \alpha \nu \sigma \varsigma, K \dot{\alpha} \nu \delta \alpha \rho \alpha$ und $K \dot{\alpha} \nu \delta \alpha \sigma \alpha$. Das erste gehört zu der grossen Sippe der antni, zu $K \dot{\alpha} v \delta a \varrho \alpha$ vgl. Cantrius (häufig) und Cantherius Berg im Sabinerland, zu Kó $\nu \delta \alpha \sigma \alpha$ Cantasius.

2) Zur Endung von Elyros vergleicht Fick eine Reihe von Namen, von denen Aósuvo unten behandelt wird, zu den übrigen hier kurz einige Parallelen: Al'ovoos

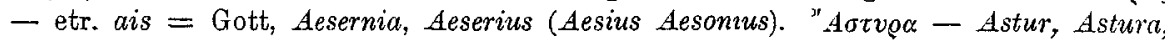
Asturius, Astrius, Asturnius, Astranius. Lipvea - limurce, limrce-, Iemrecna, lemrcna, Limbricius (Sch. S. 180 n. 1). Kißv@а - Ciprinius, cipiru, cipirunia (Sch. S. 271).

3) Dass die mit $v \vartheta$-Suffix gebildeten Wörter im Griechischen sowohl wie im Etruskischen Diminutiva sind, suchte ich in der Abhandlung Ist das Etruskische eine hettitische Sprache? I. Ueber das $\nu \vartheta$-Suffix etc. (Gelsenkirchen-1909) darzutun. 
zusammen; "schon Niebuhr hat", wie Schulze S. 573 bemerkt, "Tarraco mit den Etruskern in Verbindung gebracht".

Wir kommen zu Ficks Kapitel "Pelasger in Kreta" und wenden uns zuerst zu dem ausser Kreta auch sonst vielfach begegnenden Stadtnamen $\Lambda \alpha \dot{\rho} \iota \sigma \alpha \epsilon$ (F. S. 20).

Nach St. Byz. wurde Tóøvvv auch Láoıo genannt, dazu bemerkt Fick: "Vermutlich war es die Burg, die den Namen Larisa trug, wie die Burg von Argos ebenfalls hiess". Es liegt nahe den Namen mit dem etr. Vornamen lar und der ungeheuren Masse von Namen, die von ibm abgeleitet sind, in Beziehung zu setzen; so könnte der Name zu dem Vornamen laris gezogen werden; aber wegen des häufigen Vorkommens von Larisa in allen Gebieten vorgriechisch-hattidischer Bevöllkerung erscheint es mir noch wahrscheinlicher, dass ein etruskisches Appellativum: lar $=$ Fïrst, Herrscher zugrunde liegt, Larisa demnach etwa Herrschersitz, Fïrstensitz bedeutet. Eine passendere Bezeichnung kann schwerlich für diese Orte gefunden werden; denn es handelt sich bei ihnen durchweg um solche, die eine zentrale Lage hatten und von denen aus man die Gegend beherrschen konnte. In Campanien gab es übrigens auch ein Larisa, sodass also auch der Stadtname in Italien wiederkehrt.

$L \varepsilon \beta \dot{\eta} \nu$ oder $\Lambda \varepsilon \beta \eta \dot{v} \eta$, Hafenort von Gortyn, hielt man früher für phönizisch und brachte es mit 'לב zusammen. Dagegen bält Fick es für pelasgisch und stellt es zu $\Lambda \dot{\varepsilon} \beta a, \pi \delta \lambda_{\iota \varsigma} \dot{v} \pi \dot{o} \Theta \varrho \not \varkappa \varkappa \tilde{\omega}$, (Hes.), wo er ein Wort wie "zerstört" ergänzt" ${ }^{1}$, $\Lambda \varepsilon ́ \beta \varepsilon \delta o s$, das nach Pausanias früher den Karern gehörte, $\Lambda \varepsilon \beta \dot{\alpha} \delta \eta$, ebenfalls in Lydien, $\Lambda \varepsilon \beta \dot{\alpha} \delta \varepsilon \iota \alpha$ in Boiotien und $\Lambda \dot{\varepsilon} \beta \iota v \vartheta{ }^{\circ}$ s. Für die Richtigkeit dieser Auffassung spricht der Umstand, dass $\Lambda \dot{\varepsilon} \beta \eta_{S}$ der Vater des kretischen Wahrsagers Róxıos, der eine kretische Expedition nach Asien führte und dessen Name sicher zur Stadt Paṽzos gehört (s. unten S. 39), geheissen haben soll (Schol. zu Apoll. Rhod. I, 308). Für den hattidischen Charakter dieser Namen spricht ferner der Name des Gebirges $\Lambda \varepsilon \pi \varepsilon \dot{\varepsilon} v u v o \varsigma(F .62)$ dessen eponymer "Heros als Gemahl der Heroine Methymna galt", eine Anschauung, die insofern richtig ist, als beide Namen dasselbe Suffix $\mu \nu 0 s$ und $\mu v a$ haben, das Fick mit Recht als hattidisch betrachtet, insofern aber unrichtig ist, als, wie wir später sehen werden, $M \alpha \dot{q} \vartheta v \mu \nu a$ genau einem etruskischen männlichen Gentilnamen entspricht. Der Stamm Lep(e)t- steckt nun in dem lateinischen Cognomen Lepta, das, ausser in der Weiterbildung Leptinii, zweimal vorkommt und wohl sicher etruskisch ist; denn es erscheint einmal in Verbindung mit dem Gentilnamen Paconius, der offenbar zu einer etr. Namengruppe gehört (Sch. $203 \mathrm{f}$.). $\mathrm{Zu}$ der Form des Wortes Lepta vergleiche man nocb Ko@viлtc (genet.) in der Inschrift Smlg. 5075, das ich nicht mit Bechtel, Spitznamen S. 65,

1) Da indessen $\lambda \varepsilon \beta \alpha$ nur einmal in einem thrakischen Ortsnamen, 'A $A \circ o \lambda \dot{\beta} \beta \alpha$, vorkommt, oft dagegen $\delta \varepsilon \beta \alpha$, so ist Thomaschecks Vermutung, dass nur ein Schreib-

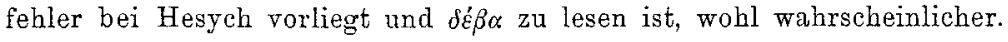




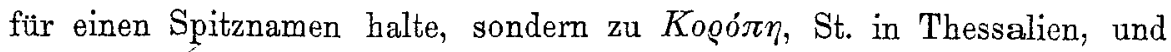

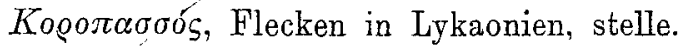

F. S. 21. Der Hauptort der Pelasger in Kreta war Tóovv(v) $)_{\text {, }}$ Tóocvv oder Toøvv́va. Der mittleren Form entspricht genau etr. * curtun, der letzteren lat. Cortona. Schulze S. 78 sagt: „Der etr. Stadtname Cortona wird in der Folge seine Identität mit dem erschlossenen curtu (curVu, curVuna) enthüllen" und S. 573 f.: "Das Verhältnis von Cremona: Cremutius wiederholt sich genau zwischen dem Stadtnamen Cortona und dem Gentilnamen curvate, in der Inschrift CIE 2470 (Clusium) largi minati curVutes. So wird deutlich, dass auch der Name Cortona uns einen sonst verschollenen Geschlechtsnamen curVu oder curvuna vertreten muss". Auf einer Inschrift aus Cortona, CIE 471, finden wir den Namen curtun-. Leider ist der Sinn der Inschrift mi unia[l] curtum nicht sicher gedeutet, vielleicht handelt es sich um ein Weihgeschenk (tinscvil steht auf dem manubrium) einer uni curtunei ${ }^{1}$ ).

Es verdient nun besondere Beachtung, dass der von Schulze erschlossene aber etruskisch nicht bezeugte Name curtu oder cur $\vartheta u$ sich direlst noch inschriftlich in Kreta findet, und zwar zunächst noch in Gortyn selber,

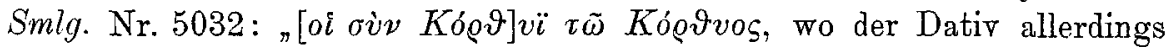
auf Konjektur beruht, der Genetir aber sicher ist; sodann in einer Inschrift aus Latos Smlg. 5079, wo ebenfalls der Genetiv Kó@きvos vorkommt. Da es sich in beiden Fällen um jüngere Inschriften bandelt, kann der Konsonantenwechsel von $\varkappa$ und $\vartheta$ gegenüber der offiziellen Schreibung mit $\gamma$ und $\tau$ nicht befremden, um so weniger, als St. Byz. von dem gleichnamigen $\Gamma o ́ g \tau v \varsigma$ in Arkadien berichtet, dass es auch Kóovvৎ und danach

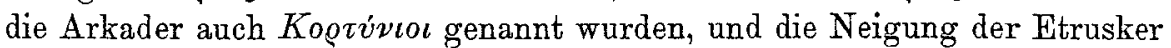
zur Aspiration der Mutae sattsam bekannt ist, wie ebenso die Griechen bei Fremdwörtern gern die Aspirata setzen. Der Name Kó@qvs ist identisch mit dem des Heros Tóovv, nach welchem St. Byz. zufolge der Ort benannt sein soll und auch tatsächlich benannt worden sein wird, abgesehen davon, dass ihm das griechische Nominativ-s fehlte; Kó@v $v_{\varsigma}$ wird also nicht, wie Bechtel (1. c. S. 40) meint, ein Spitzname sein; dasselbe gilt auch von dem gleich zu behandelnden Kvं $\lambda \lambda o s$.

Die Inschriften von Gortyn bieten noch mehrere andere Namen, die zu spezifisch etruskischen zu gehören seheinen:

Der Name Ká@avos (Smlg. 5016) kehrt wieder in Caranius, das aber

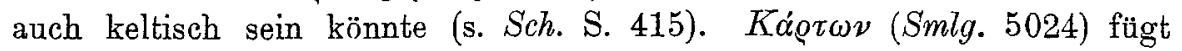
sich einer etruskisehen von Sch. unter Cardenus aufgeführten Gruppe organisch ein. Smlg. $5028 \mathrm{~B}$ wird ein $\Delta \iota \sigma \grave{s} \varsigma \Lambda v \delta \alpha u ́ u \iota \varsigma$ aus Halikarnass zum Proxenos von Gortyn erklärt, er dürfte zu Disins, Disinius, Tisenius, etc. gehören. In der Insehrift 4991 finden wir eine Gottheit Toxoi $\alpha$, sie

1) Vgl. Sch. S. 364 . 
erinnert an Tucca, Tuccius, Tucasius tucuntual (Sch. S. 375), einen $K \dot{v} \lambda \lambda$ s

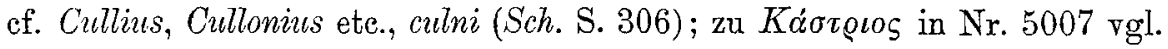

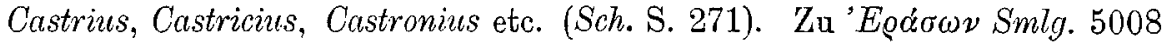
gehört wohl Erasenus CIL VI 20938 (Sch. 344); da der Name ziemlich vereinzelt dasteht und es zweifelhaft ist, ob die von Schulze mit ihm zusammengestellten wirklich $\mathrm{zu}$ ihm gebören, so kann es sich natürlich um einen vereinzelten Kreter handeln, der in Rom eingewandert ist. Smlg. 5030 (vom Pythion in Gortyn) lehrt uns einen $K \dot{\alpha} \lambda \alpha \beta \iota \varsigma$ kennen. Bechtel

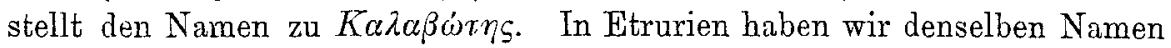
als calapi, er gehört der zweifellos altetr. Familie der petru calapi in Clusium an. Schulze (S. 138) erklärt es für zweifelhaft, ob calapi verwandt ist mit Calpennia, Calpenius, Calpurnius etc., hält es dagegen für wahrscheinlich, dass Calabius $=$ Calavius, wie eine Familie in Capua hiess; jedenfalls gibt es im Griechischen von dem oben erwähnten $x \alpha \lambda \alpha \beta \dot{\omega} \tau \eta \varsigma$ abgesehen nichts, was mit dem Namen in Verbindung gebracht werden

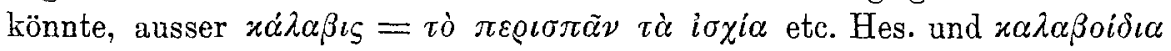

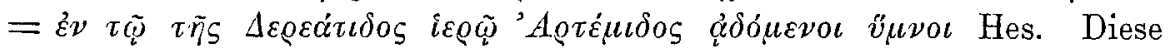
beiden Wörter scheinen aber nur Varianten gleichbedeutender Wörter mit $o$ statt $\alpha$ in der ersten Silbe zu sein und zu $\chi 0 \lambda \tilde{\alpha} \cdot \varepsilon \tilde{i} \delta \circ \varsigma$ $\partial \varrho \chi \dot{\eta} \sigma \varepsilon \omega \varsigma$ etc. Hes. zu gehören, das Thomaschek ${ }^{1}$ ) von der Wurzel „q'el: qol- sich drehen, sich beweǵen " ableitet.

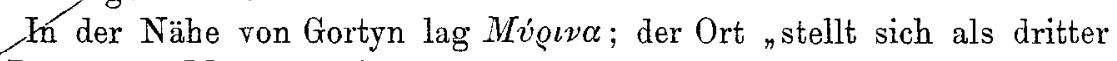
im Bunde zu Mv́oıva auf Lemnos und in der Aeolis". Der Name erscheint auch in der vorgriechischen Inschrift von Lemnos in der Form morinail. In Mv́@ıva haben wir direkt ein etruskisches Gentilicium vor uns, das vielfach im CIE als murina oder Murina oder in lateinischer Uebertragung als Murrenius im 11. Bande des CIL vorkommt. Daneben gibt es muria, lat. Myrrhius oder Murrius, murunial = lat. Mutronius nebst zahlreichen anderen Verwandten. Schulze vermutet (S. 195), dass auch lat. Murena nur eine Variante von murina sei und mit dem Fisch murena nichts zu tun habe.

F. S. 22: "Nordwestlich von Gortyn lag $\Sigma \dot{v} \beta \varrho \iota t \alpha$ (Münzen $\Sigma v \beta \varrho i-$ $\tau \iota 0 \nu^{\prime \prime)}$, das nicht bloss in Attika, sondern auch in Illyrien Verwandte hat. Die Form $\Sigma v \beta \varrho i ́ \tau \iota v \nu$ ist gräzisiert, die einheimische Form wird $\Sigma \dot{v} \beta \varrho \iota \tau \alpha$ gewesen sein, das eine vollständig etruskische Gentilicialform trägt. Das Suffix iๆ $a$, ita oder $i \vartheta e$, ite ist in etruskischen Namen sehr häufig, ich stelle aus dem etruskiscben Index bei Schulze folgende Formen zusammen:

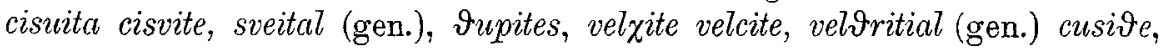

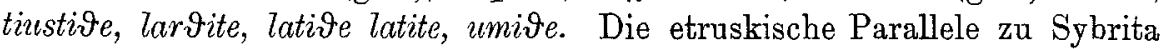
ist das forum Subertanum in Etrurien; die Form, aus der sich mit obigem Suffix Sybrita entwickelt hat, liegt vor in zupre CIE 2251 (Clusium), supri CIE 53 (Volaterrae) = lat. Subrius CIL V 7917 u. ö. und Sobrius,

1) Die alten Thraker II, 14. 
mit gentilizischem $n$ : Subernius. Die auch vorkommende Nebenform $\Sigma o v$ $\beta \varrho \imath \tau \alpha$ zeigt, dass gr. $v=$ lat. $u$ zu sprechen ist.

„Wiederum nordwestlich von. Sybrita wird die Lage der alten Stadt $B \eta \dot{\nu} \eta$ durch das heutige Dorf Veni verbürgt." Durch Vergleichung von

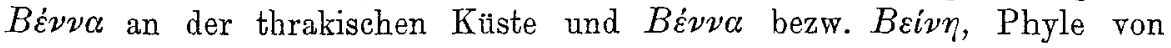
Ephesos, kommt Fick zu dem Schluss, dass BévFos die ursprüngliche Form sei. Dazu werden gehören Benus CIL VI 18961 (Sch. 133 unter Biennius), Be(n)nius, das häufig vorkommt (Sch. 423), Pennus (Konsul 167, Sch. 365), ferner die nur in Etrurien inschriftlich erscheinenden Pennasius (CIL XI 5115), Penasius (5053 Mevania), Pensius (XI 5386 Asisium) und penףe (CIL 3048 Clusium).

Aus $B \dot{\nu} \nu \eta$ stammt der Dichter 'Pıavós, auf dessen nicht griechische Endung Fick S. 23 hinweist. Den Namen dieses Dichters haben wir jetzt inschriftlich in doppelter Form, und zwar auf einer Stele Smlg. 5124,

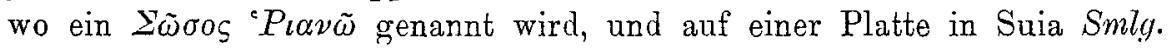

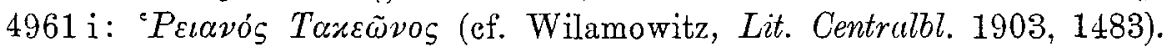
Genau dieselbe Form wie die letztere, Reianus, finden wir CIL VI $25391 \mathrm{ff.}$., ferner reisni CIE 342, Raius (CIL XI 4914 Spoletium) sowie Raienus und

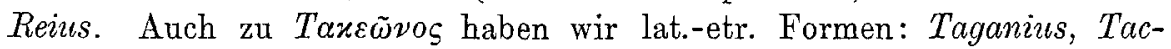
cianorum etc.

Von den Orten, die Fick den Eteokretern zuweist (S. 23 f.), ist oben S. 27

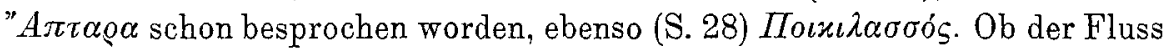
$M \varepsilon \sigma \sigma a \dot{\pi} \iota{ }^{\prime}$ s kretisch ist, wage ich nicht zu entscheiden, dagegen muss ich

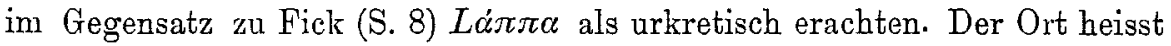
bei St. Byz. auch $\Lambda \alpha_{\alpha}^{\prime} u \pi \eta$, und dies hält Fick für die ursprüngliche und damit griechische Form. Dafür spricht allerdings, dass gleichnamige Städte in Arkarnanien und in der Argolis existierten; dagegen zeigen die In-

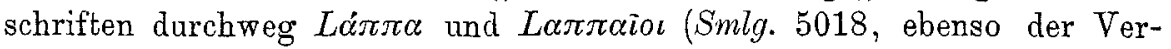
trag der Kreter mit Eumenes, ferner Smlg. 5075a).

Sehr merkwürdig ist nun, dass wir in Italien den Namen Lappa als Cognomen finden und eine Reihe von Weiterbildungen desselben Namens

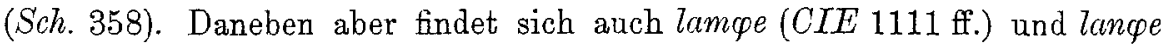
und lape, lat. Lamponius. Es scheint mir wahrscheinlich, dass wir die ursprüngliche Form in Lappa zu suchen haben, welches sowohl Stadtname als Familienname war, und dass der Name auf kretischem und ebenso auf italienischem Boden auch nasaliert wurde.

Für den Wechsel von $\mu \pi$ und $\pi \pi$ vergleicht Fick S. $9 \lambda \dot{\alpha} \pi \eta=\lambda \dot{\alpha} \mu \pi \eta$ "Schimmel, Kahm" bei Hes.; als Beispiel dafür, dass die Griechen sich doppeiten Dental fremder Sprache durch Verwandlung des ersten in $\mu$ mundgerecht machten, verweise ich auf $\sigma \alpha \mu \beta \dot{v} x \eta$, das aus dem aramäischen sabbeka stammt.

Zum Namen Kóoıov (F. S. 24) gehört der in der Nähe gelegene

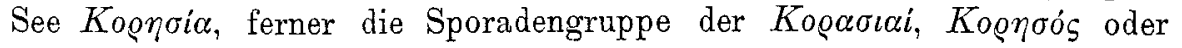




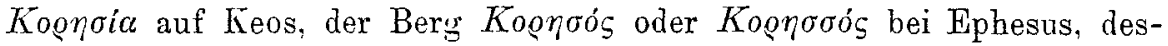
sen eponymer Heros als Autochthon bezeichnet wird; weiter rechne ich hierher Kooviv in Elis, Koøvंva, Stadt der erythräischen Halbinsel, Vorgeb. Koovvvaiov, die wohl mit gr. xooviv $=$ Keule nichts zu tun

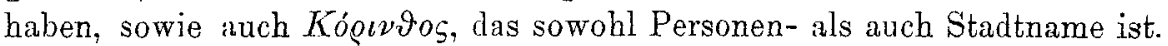
Diese ganze Gruppe kehrt in Etrurien und in lateinischen Namen wieder,

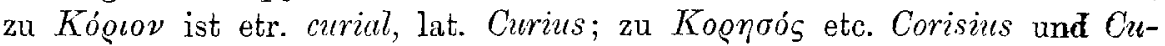
risizus, zu Ko@ivin etr. curuna, zu dem Deminutiv Kóouvos Corioli die entsprechende Form. Auch die Curiatii sowie die volskisehen Städte Cora und Corella dürften hierher gehören. Eine Nebenform von Ko@v́v ist

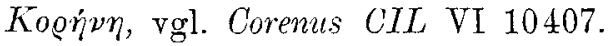

$\mathrm{Zu}$ dieser Gruppe gehören auch die Gentilnamen Coronc und Coronius = etr. cumuna und zurua. Auch diese Formen finden ihre Analogie in Kreta und zwar in der Inschrift Smlg. 5015, wo es heisst: $K \nu \omega \sigma o \tilde{\iota} \delta \dot{\varepsilon}$ etc. $\pi \circ o$

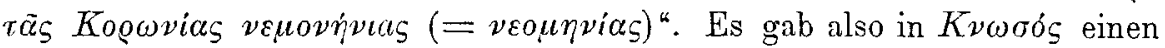

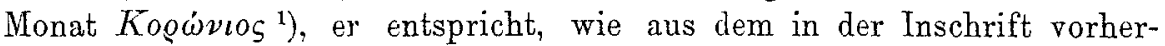

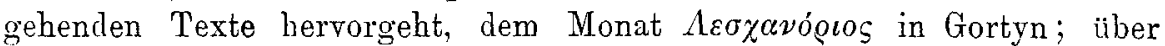
diesen Monat Ko@úvıos ist mir nichts weiter bekannt, es liegt jedoch die

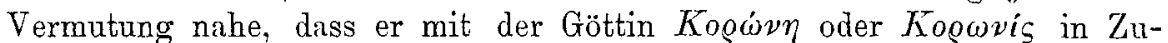
sammenhang steht, die zum Stamme der Phlegyer gehört, der wahrseheinlich hattidisch ist. Dass in der Sage von den Ko@wvides bei Ovid zwei männliche Coronce auftreten statt weiblicher, hat man als arge Verwirrung bei ihm bezeichnet; es erklärt sich, wenn wir wissen, dass corona eben ein

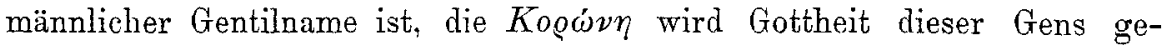
wesen sein, wie Voltumna und Vertumnus etruskische Gentil-Götter waren. Da bei den Etruskern sehr häufig eine männliche und eine weibliche Gottheit neben einander standen, wie Cacus und Caca, so wird es vielleicht auch eine männliche und eine weibliche Gottheit Corona gegeben haben. So müssen uns die etruskischen Namen die griechische Mythologie erklären helfen.

$\mathrm{Zu}$ der von Fick (S. 24) ferner behandelten Namengruppe vom Stamm Arb- stelle ich folgende vorgriechische und etr. bezw. lateinische Namen gegenüber:

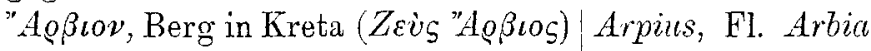

"Aoßa, Ort in Achaia Arpi in Apulien, Arbaianus

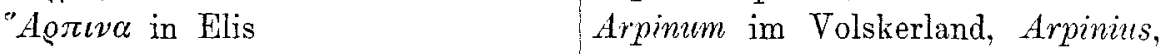
Arpinianus

'Aoßivvas P.N. Lykien Arbenizus 'Aoßvidns P.N. Karien

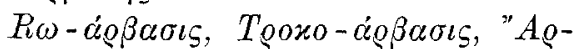
Arbula, Vater des Vinius Etruseus $\beta \eta \sigma \sigma o s$ P. N. (letzterer vom Orts-

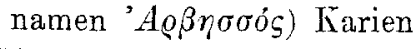

1) Derselbe Monat heisst allerdings in einer Inschrift von Delos, Smlg. 5149, la@úvıs, doch wird jene Form wohl die richtige sein. Kllo, Bertrage zur alten Geschichte XI 1. 
Im Gebiete von Arpinum lag Sora, das ebenfalls etruskisch ist und

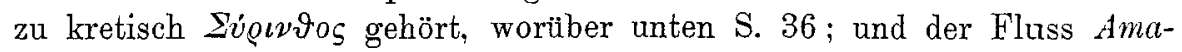
senus, noch heute Amaseno, gehört zu Amatius Amantius in Clusium, Amantinius in Florenz (Sch. S. 345, 121 und besonders 572). In Bruttium gab es eine Stadt Amantia. welche wohl der epirotischen Landschaft

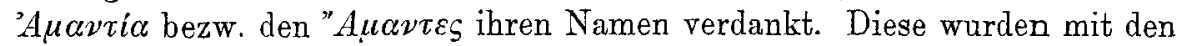
"A $A \alpha \nu \tau \varepsilon S$ auf Euboea identifiziert und Fick hält diese für Leleger; aber nach dem Zeugnis des Thukydides sind die Abanten Thraker, es bleibt daher das bruttische Amantic hier lieber ausser Betracht, da vielleicht nur ein zufälliger Gleichklang mit Namen etruskischen Ursprungs vorliegt, auch dürfte der Amasenus mit 'A $\mu \alpha \dot{\sigma} \sigma \varepsilon \alpha$, Stadt in Pontos, verwandt sein. Im Gebiete von Arpinum lag ferner Cereatae (Marianae), dies ist, wie auch weiter unten (S. 35 f.) erhärtet werden soll, ebenfalls etruskiseh und mit Kai in Kreta verwandt. Ich führe alles dies schon hier an, um jeden Zweifel an dem etr. Ursprunge von Arpinum zu beseitigen. Die Könige, die dort einst geherrscht haben sollen, sind ein etruskisches Adelsgeschlecht, das

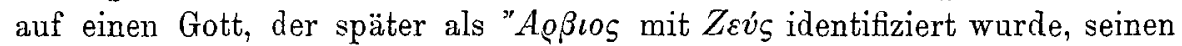
Ursprung zurückführte.

F. S. 25. Der Fluss- und Stadtname 'A

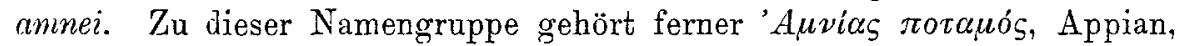

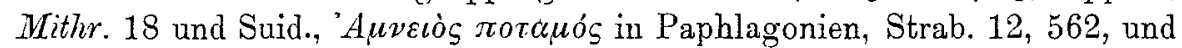

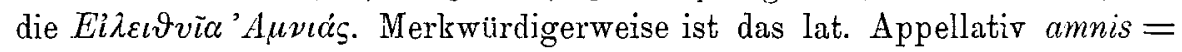
Strom ganz arm an Weiterbildungen. Es liegt daher die Vermutung nahe, dass amnis ein etruskisches Lehnwort ist, in welchem zugleich der Name einer Gottheit steckt, die mit der Eileithyia verbunden wurde, weil sie eine Gottheit der Zeugungskraft und der Fruchtbarkeit war.

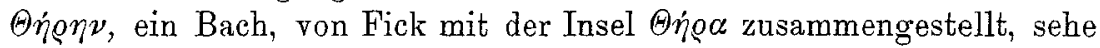
ich als gentilizisches Adjektiv an wie Sarmus, das den Bach als der gens * $\Theta \dot{\eta} \varrho \alpha$ oder * $\Theta \eta \emptyset \dot{v} \nu \alpha$ angehörig bezeichnet, dann haben wir diese gens vielleicht in etr. Veras' (Sch. S. 373), lat. Terius (Sch. S. 68) u. a.

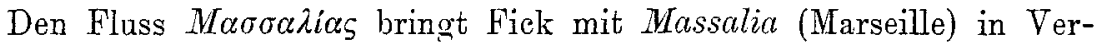

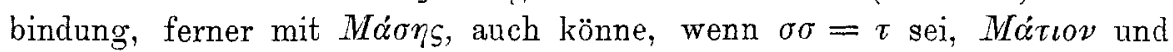
$M \alpha \dot{\tau} \alpha \lambda \alpha(o v)$ dazu gehören. Kreter würden Massalia schon vor den Phokäern kolonisiert haben.

Für die vom Stamme Mas- bezw. Mass- gebildeten Namen tritt allerdings keltische Konkurrenz ein, aber es gab in Etrurien mehrere Orte, die Massa hiessen ${ }^{1}$ ) und die jedenfalls etruskisch sind; auch der Mons Massicus im Aurunkerlande wird einen etruskischen Namen haben, ebenso die mO Massignano bei Ancona und Massiano bei Perusia. Zablreiche Personennamen von diesem Stamme haben wir in etr. sowohl wie in lat Inschriften; sie bieten die Formen masni, masnial, masu, masui, Masonins, Masius, Masidius, Masidianus, Massidius, Masurius, und was für unsere

1) Nissen, It. L. II, 306. 


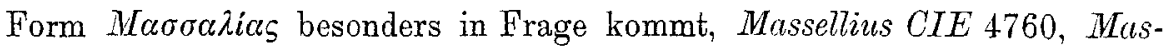
sillius CIL XI 4758 (Tuder), mslnei CIE 3570,3789 ff., maslni 4394 (Sch. 68n, 278, 373).

Schliessen wir hier gleich Márıov, Küstenort westlich von Knosos,

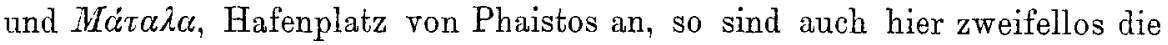
etruskischen Parallelen vorhanden und kaum Zweifel möglich, dass die entsprechenden Namen altetruskisches Sprachgut sind, das Material ist bei Schulze, S. 274 f.; es sind: Matinius, Matenianus, Matho, mutuna, Matomins, fundus Maticianus, Maticius, verbreitet sind Matius (mit Matidius, Matienus und Mattius), nur vereinzelt begegnen Matianius und Matisius,

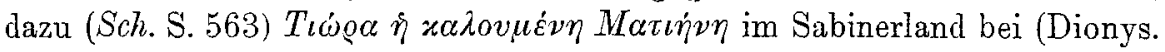
Hal. antiqu. I, 14), dessen „Beiname offenbar mit der gens Matiena zu-

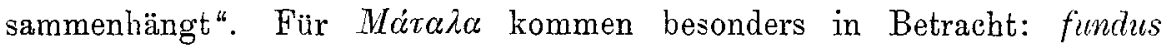
Matellianus in Veleia, "Matellius ist selten“, Matlia, nur in Praeneste, matulna (Tarquinii), mavl-(Clusium); dazu kommt der Ort Matilica in Umbrien (Sch. S. 552). Auch Mávvuva auf Lesbos dürfte dieser Gruppe nicht fremd sein, da, wie wir gleich sehen werden, die Endung mna ebenso gut etruskischen wie vorgriechischen Namen eigen ist. Auf die merkwürdige Tatsache, dass der Name des römischen Konsuls Matienus wieder-

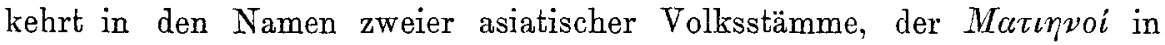
Kappadokien und in Medien, hat Wirth schon hingewiesen ${ }^{1}$ ). Es ist kaum zu bezweifeln, dass diese Stämme auch hattidisch sind; die ersteren gehören zu den Bewohnern Kappadokiens, die vor der phrygischen und galatischen Einwanderung schon dort ansässig waren, die letateren nennt Herodot III, 94 mit Saspiren und Alarodiern zusammen als dem 17. Kreise des persisehen Reiches angehörig.

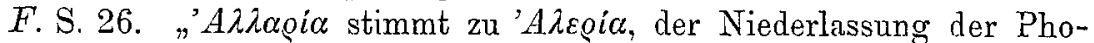
käer in Corsica". Es ist bekannt, dass die Etrusker Niederlassungen auf Corsica hatten, sie werden dort wie in Massilia den Phokäern vorangeyangen sein, bezw. eben Kreter den Ort gegründet haben. Auf italischem Boden begegnen wir sowohl Alarius als Alalius, doch stammen beide Inschriften nicht aus Etrurien.

Der Fluss 'A yraias bei Priansos und der Name des Lelegerkönigs 'A yraios kann zu Ancus gehören und der Gruppe Anquirinnius bei Schulze S. 122.

Kaioatos, der alte Name für Knossos, wohl mit dem etr. Suffix ate gebildet, gehört zu Caere. „Der maskulinische Nominativ zu zaireals kann zaire gelautet haben, das ist zugleich der Name der Stadt Caere", sagt Schulze S. 567, und S. 354 sagt er: "In Etrurien hat es ein Geschlecht der zaire oder zairea gegeben". Danach ist Kaipatos der Ort, der der Familie Kaire gehört, Kaịatos auch der ihr gehörige Fluss wie Surnus

1) Politisch-anthrop. Revue, VI S. 200. 
der Fluss der Sarii ist. Zu Caere gehört das Geschlecht der xeritna, das in Saena und Clusium vorkommt (Sch. S. 529).

Wie hier altes $a i$ (über $e i$ ) zu $e$ geworden ist, darf der gleiche Vorgang auch bei Cereatae angenommen werden, das oben (S. 34) schon erwähnt ist. Das vor dem Suffix at vorgeschlagene $e$ hat sein Analogon in Gereonium $=$ Gerunizim.

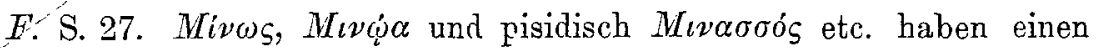
in Etrurien mehrfach vorkommenden Stamm, zahlreich ist die Familie der minate, lat. Minatins, ferner Minacius, Minasius, Minius, Minidius; etruskisch ist auch Minturnae in Aurunkerland.

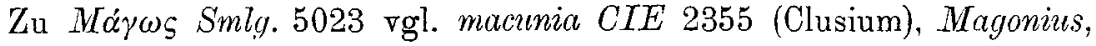

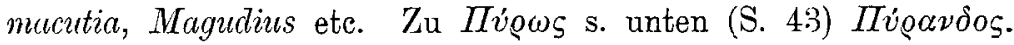

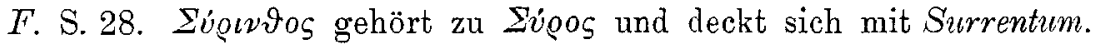
Der Name gehört einer sehr weit verzweigten etruskischen Namengruppe an, die bei Schulze S. 235 und 271 verzeichnet ist; die einfachste Form ist sure, welches gr. Ev́oos entspricht (Kyklade und Insel bei Akarnanien). Das in Etrurien mebrfach vorkommende surna entspricht genau $\Sigma v$ ov Stadt in Karien. Diese Stadt wird auch $\Sigma$ v́ovos genannt, genau wie eine der Kykladen heisst; die lateinische Form des Gentiliciums surna ist Sornius. Diese Namengruppe lebt fort in mehreren Ortsnamen: Sora im Volkskerlande, das schon oben (S. 34) als etruskisch genannt wurde, Sorgnano (findus Sornianus in Veleia), Soriano, Sorcicno.

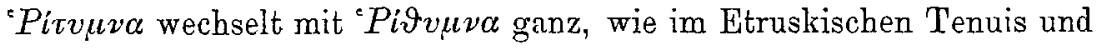
Aspirata unzähligemal wechseln, z. B. in ratumsna neben raףumsnal. Die in Kleinasien häufige Endung $\mu \nu \alpha$ kommt auch in etruskischen Namen vielfach vor, bisweilen steht noch ein $e$ zwischen $m n$, dies ist nur ein eingeschobener Vokal, die richtige etruskische Form wird vokallos gewesen sein. Bisweilen wird auch ein $s$ eingeschoben, so steht neben obigem ratumsna: Ratumennia, neben arcumsnei: Arcumenna. Für die Häufigkeit solcher Namen im Etruskischen genügt folgende Zusammenstellung von rein etruskischen Formen aus dem Index bei Schulze: arcumsnei, clumnei,

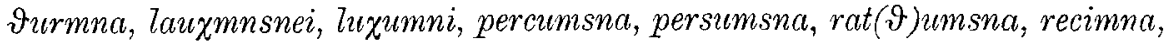
restumnei, rems(z)na, ucumzna, $f(h)$ elzumnati (heizumnatial), malamenas, sehtmnal, velimna, pustminas, tucmenas, sesumsnei, setumnei, tarchumenaia, ultimne, utimnal.

Dem Namen 'Pitvuva entspricht nun genau etr. ritumenas. Wir haben es hier mit einem so eigenartigen Lautgebilde zu tun, dass seine Wiederkehr auf zwei verschiedenen Gebieten kaum als Zufall angesehen werden kann, umsoweniger als noch eine zweite kretische Stadt ron dem näm-

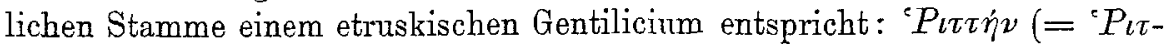

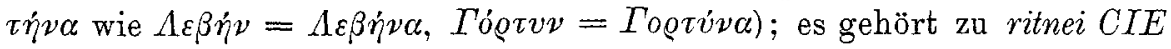
1616 (Clusium), es stimmt dazu auch lat. Ridlanius. Zweifelhaft ist, ob auch Retinius, Retilius und Retonius etruskisch sind (s. Sch. 278 n. 1). 
Diese Namen setzen eine Grundform rite voraus, welche wir in Fabr. 802 und 803 finden; da indessen der Sinn dieser Inschriften nicht klar ist, ist es nicht unmöglich, darin mit Bugge, Fo. und Stud. IV, $44 \mathrm{f}$. ein Verbum zu sehen. Bugge betrachtet auch rivce (Fabr. 2596) als Verbalform, es kann jedoch auch ein Name sein, gebildet wie larce.

Was nun die Form 'Pıtrív betrifft, so gibt St. Byz. dieselbe als

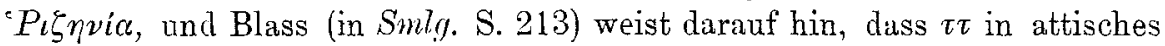
$\sigma$, aber nicht in $\zeta$ umgesetat werden müsste. Es wird daher wohl 'Pıt$\tau \dot{\eta} v$ nicht einfach $=$ etr. ${ }^{*}$ riten bezw. ${ }^{*}$ ritenna sein, sondern eine $s$-Bildung wie versena vorliegen, so dass * ${ }^{*}$ Pı $\tau \eta^{\nu} \nu$ die Ausgangsform ist, aus der teils ${ }^{\circ} P_{\imath} \imath \tau \dot{\eta} \nu$ teils ${ }^{'} P_{\iota} \zeta \eta^{\prime} \nu$ wurde. Der Stamm des Wortes ist rit, da-

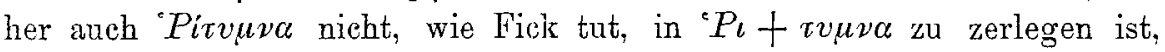
sondern in ${ }^{\circ} \mathrm{P} \iota \tau+u+\mu \nu a$ oder, da Ritu ein fertiges etr. Gentilicium dar-

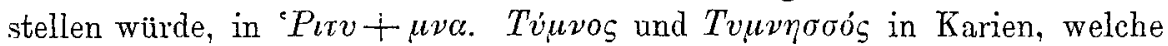
Fick zum zweiten Teil von Ri-tymnu vergleicht, haben Tvu-als Stamm und das etr. gentiliciabildende $\boldsymbol{\nu}$. Sie gehören zu dem kretischen P.N. Tv́$\mu \omega \nu$, der bei dieser Gelegenheit gleich mitbehandelt werden mag. Der Name kommt mehrere Male inschriftlich vor: Smlg. 5056, 5076 und 5078. Er entspricht genau etruskisch tumu CIN 2997-3000 (Clusium). Das karische Túuvos entspricht genau etr. tumumias CIE 2600. Obwohl nun von dem Stamme tum- weitere etruskische oder lateinische Namen nicht vorkommen als die genannten, so ist der etruskische Ursprung derselben doch um so sicherer, als lateinische oder den Italikern angehörige Namen, die nach Etrurien gewandert sein könnten, nicht vorhanden zu sein scheinen und der Sohn jener Tumunia, der Träger der eben genannten Inschrift, ein presnte ist und zu der an Moaioos sich anschliessenden Gruppe gehört (s. ı. S. 44).

Von den andern hattidischen Namen mit dem Element $\mu \nu \alpha$, welche Fick

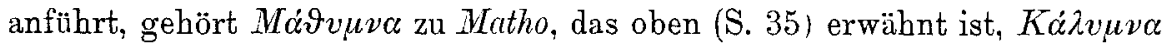
kehrt wieder in clumnei CIE 4305 (Perusia), dis wohl zu calunc (welches ausser den von Sehulze S. 171 angeführten Inschriften von Clusium auch in Perusia CIE 4305 bezeugt ist), ebenso wie auch Klum. in der oskischen Inschrift Conway Nr. 121 gehören wird. Calumeins, Calmeius, Calmaeus entsprechen etr. ${ }^{*}$ calumna, wir sehen also in Kádvuva direkt die Form eines etr. Gentiliciums. Die ganze etruskische Namengruppe (zu der auch der Gott calu gehört), sehe man bei Schulze S. 251/2.

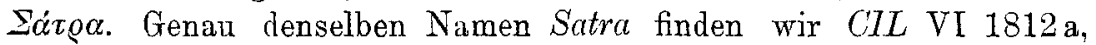
satrial in Clusium (CIE 2395), die zngehörige Namengruppe ist in Italien weit verbreitet; Schulze bemerkt S. 225, wo sie eingehend behandelt ist, dass Satrenus, Satrenius, Satrinius, Satronius, etruskischer Herkunft einigermassen verdächtig sind, da das freilich auch sonst in Italien häufige $S a$ trius in Etrurien ganz eingebürgert ist". Auch die Verbreitung der nFormen sei der Annahme nicht ungünstig, dass ein etr. satrna zugrunde liege, wie denn auch saternas aus Volsinii belegt sei. Im modernen Orts- 
namen Satriano lebt diese Namengruppe fort, zu der vielleicht auch $S a$ tricum gehört. Fick vermutet, dass $\Sigma \dot{\alpha} \tau \varrho \alpha=\Sigma \alpha \dot{\alpha}$. $\alpha$ ar $\varrho \alpha$ sei und sich mit $\Sigma \alpha v \alpha \tau \varrho \alpha, \Sigma \alpha \dot{\beta} \alpha \tau \varrho \alpha$ in Isaurien decke. Merkwürdigerweise haben wir in Etrurien neben sature CIE 2736 (Clusium) und saturinies, Fabr. s. 3, Gam. 745 (Viterbo), auch sauturine, sauturini, scututrinial, sautri, zauturia, so dass diese etruskischen Formen aus den kretisch-kleinasiatischen Namensformen ihre Erklärung finden; hierin darf gewiss wieder ein unumstösslicher Beweis für die kretisch-kleinasiatische Herkunft der Etrusker geseben werden.

Ein Digamma, das bier in kretisch $\Sigma \alpha^{\prime} \tau \varrho \alpha$ verloren gegangen ist, hat sich

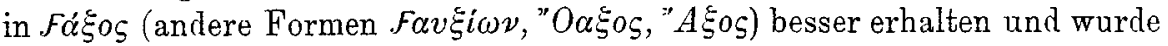
von den Vaxiern in ihren Insehriften bis in späte Zeit hinein treu festgehalten. Den nichtgriechischen Charakter des Namens zeigt schon die Vielheit der Transskription an. Der Name kann in Baxius, pacsneal, pacsnial, pacsinial wiederlzehren und in der dazu gehörigen Namengruppe (Sch. S. 213).

Der Hafen vor $f a \dot{\xi} \xi o \varsigma$ war wahrscheinlich 'A $A \tau \dot{\alpha} \lambda \eta$, einen Aminnas Astlas haben wir CIL VI 647; doch ist die Ueberlieferung nicht sicher und vielleicht Asclas zu lesen, jedenfalls kann 'A $A \tau \dot{\alpha} \lambda \eta$ zu astnei (CIE 52) und zu dem öfter vorkommenden Astius gehören (Sch. S. 131).

F. S. 29. Kvंrc oder Kítatov. Die zweite Form ist Adjelztiv der ersteren. In Kvंta steckt wahrscheinlich sehon ein Gentilname, und es widerstrebte den Griechen, diesen direkt als Ortsnamen zu gebrauchen. Der Name fügt sich der unter Cotinna bei Schulze S. 78 f. zusammengestellten Gruppe organiseh ein; in cutus ist die $u$-Form des Gentilnamens vorhanden, zu welcher cutunas, cutunial, Cotonia (Pernsia) gehören, aber wir haben auch Cotena in Falerii, Cotinius CIL III 5625, Cutina im Vestinerlande und endlich cutanasa, welches die Form cuta zur Voraussetzung hat, wofern nicht das a eingeschoben ist und die richtige etr. Form vokallos war wie cutni, cutna, cuฑnai. Jedenfalls könnte cuta ohne weiteres als etr. Gentilname fungieren; die modernen Ortsnamen Cottignola bei Faventia und Cutignano bei Nola sind Ueberbleibsel dieser Namengruppe, zu der mir anch Kvjwvic zu gehören scheint, das sich mit Cotonia genau deckt. Von dem Geschlechte der cutuna $=K \dot{v} \delta \omega \nu \varepsilon \varsigma$ erhielt zuerst die Stadt, dann die Landschaft ihren Namen. Die Schwierigkeit. $K \dot{v} \tau \alpha$ mit $K v \delta \omega v i \alpha$ zusammenzubringen, die in in der Lautstufe des Dentals steckt, darf nicht zu sehr ins Gewicht fallen. Die Etrusker sprachen wie noch heute die Kaukasier alle Laute möglichst hinten in der Kehle, wodurch die Unterscheidung der Lautstufen erschwert wurde; dass die Kreter ebenso sprachen, geht daraus hervor, dass in mehreren Namen sich ein Schwanken zwischen Tenuis und Media oder Aspirata zeigt: oben (S. 30)

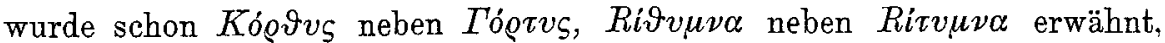

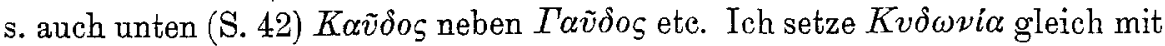

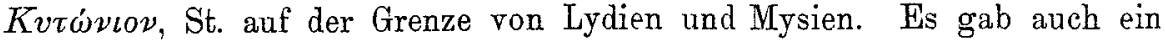

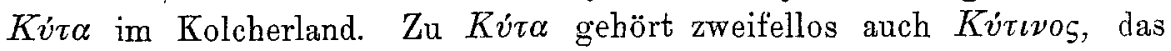


auf einem Stein in Itanos steht (Halbherr Nr. 19); mit Cutina im Ve-

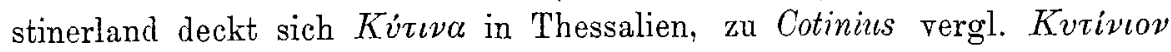
in Doris.

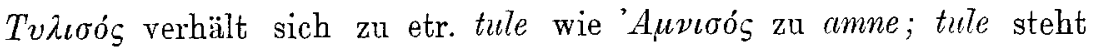
CIE 376 und 433 ; dass die letztere Inschrift alt ist, lebrt die dort vorkommende Form cavinei (Frau des tule) für späteres cainci. tule ist $=$ Tullius, welches auf etruskischem Gebiete in Faesulae, Volsinii, Viterbo und Falerii begegnet. Tullenus kommt CIL VI 6658, Tullonius in Benevent und Tullieness in Cupra Montana und Cupra Maritima vor. Es kann kein Zweifel sein, dass wir es in Tullius mit einem Gentilnamen etruskischer Herkunft zu tun haben, und den M. Tullins Cicero wird man um so eher als Etrusker ansehen, als auch seine Heimat, wie wir oben (S. 33) sahen, einen etruskischen Namen trägt und auch sein Cognomen nichts anderes als eine latinisierte Form eines etr. *cicru zu sein scheint, auf welches die Formen cicu, Cicrius, Cicereius führen und anf dessen mögliche Gleichsetzung mit Cicero Schulze zweimal (S. 273 und 315) hingewiesen hat. Es gab allerdings auch noch eine dem Cicero nicht verwandte (illyrisch-venetische) gens Tullia (Sch. S. 30 n), wie Cicero selbst gelegentlich erwähnt.

Für die Sicherheit des etr. Ursprungs von tule darf noch auf das in den Agramer Mumienbinden vielfach vorkommende Wort tul, auch auf

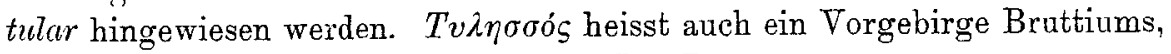
$T \hat{\lambda} \lambda \omega \nu$ ein alter lydischer König bei Nic. Dam.

Den Namen 'Paṽxos bringt Fick mit dem des Dichters 'Páxuos in Verbindung unter Berufung darauf, dass neben $F \dot{f} \xi o \varsigma$ auch $F \alpha v \xi i \omega v$, karisch $A \alpha \dot{\beta} \varrho \alpha v \nu \delta \alpha$ neben $\Lambda \alpha \dot{\beta} \varrho \alpha \nu \delta a$ stehe. Wir können noch einen Schritt weiter

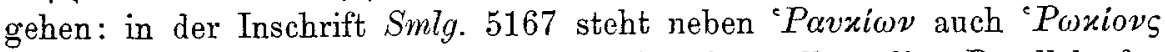
für die Bewohner von 'Paṽros. Damit ist eine vollständige Parallele für die etruskische Vokalisation gegeben; dass $a$ und au nebeneinander vorkommen, ist sehr häufig, wie wir oben (S. 38) schon saturine neben sauturine notiert haben; für den nämlichen Volal tritt aber auch $\imath=\mathrm{gr} . \omega$ ein; dies ist z. B. der Fall bei der Familie Rafia in Perusia, deren Mitglieder in derselben Grabkammer bald rafi, bald raufi, bald rufi genannt werden.

Wenn man hüben in Perusia ebenso wie drüben in Kreta bei der schriftlichen Fixierung der Laute in gleicher Weise schwankte, so müssen die Organe, mit welchen dieselben gesprochen wurden, auch die gleiche Struktur gehabt haben. Da die Kreter, wie schon oben gesagt, die Lautstufen der Mutae fast gar nicht unterscheiden konnten, werden wir kein Bedenken tragen, neben kretisch 'Póxıos etr.-lat. Ragius CIL 5915 (Anagnia), Ragaiena (VI 25 352) und Ragonius (V 3725 Verona) zu stellen; über den letzteren, der L. Ragonius Urinatius Larcius Quintianus heisst, bemerkt Scbulze S. 87 n, dass ihn, wenigstens in ethnographischem Sinne, das Zusammentreffen von Larcius und Urinatizs lokalisiert; wir haben in ihm einen echten Etrusker vor uns. 
$\Theta \varepsilon \nu \alpha i$ bei Lyktos ist zwar in altetruskischen Namen nicht vertreten, aber T'enetius und Tenatius tragen ein durchaus etr. Gepräge, einer des letzteren Namens stammt ans Ateste, ein anderer aus Verona, aus letzterer Stadt auch ein Tenagino; Tenacius begegnet dreimal, Tenatianus und Tenneius je einmal.

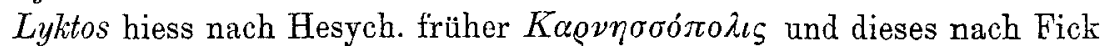

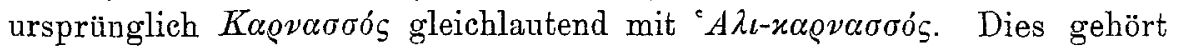
zu der Gruppe etr. carna, die man bei Schulze S. 146 und 306 zusammengestellt findet.

Midaros ist $=$ Milasius CIL VI 2662 u. ö., Miliasinss XI 1878 (Pisae), Milassius XI 6683 (Umbrien); vgl. milaei CIE 234 (Saena); Milonius, Millenius, Milienius und besonders das bekannte Cognomen Milo ${ }^{1}$ ).

"Aøñoos ist eigenartig gebildet", doch entspricht ihm genau der Fluss Trerus fl. Sacco oder Tolero im Hernikerlande, das ebenso wie das Volskerland von etruskischen Namen wimmelt. Nissen, Ital. L. S. 647, tritt allerdings für Nibbys Vermutung ein, dass Trerus nur für Tolerns verschrieben sei; da indessen $l$ und $n$ ebenso im Etruskischen häufig wechselt (vgl. ruze neben nuzlyne in den Mumienbinden VIII 14, aұmemrun = 'A $\gamma \alpha \mu \varepsilon \dot{\varepsilon} \mu \omega \nu$ ), ebenso wie in den griechischen Namen hattidischen Ursprungs (z. B. $T \varepsilon \lambda \mu \eta \sigma \sigma o ́ \varsigma=T \varepsilon Q \mu \eta \sigma \sigma o ́ \varsigma$ ), so kann Tolerus mit eingeschobenem $o$ sehr wohl eine - vielleicht auch infolge von Dissimilation eingetretene - jüngere und Trcrus die echt etruskische Form sein. Nicht unerwähnt darf indessen bleiben, dass es auch ein thrakisches Volk Toñoo gab und die Frage, ob Thraker nicht auch früh nach Kreta gelsommen sind, noch nicht entschieden ist.

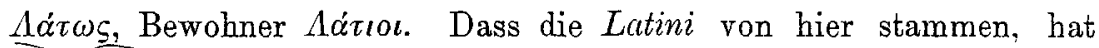
schon Wirth vermutet (Polit.-Anthrop. Revue VI 207); es erscheint aber im höchsten Grade wahrscheinlich, dass wir auch den Hafen von Latos

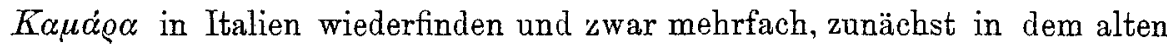
Namen von Clusium, der Camars lautete, auf Münzen Cha abgekürzt ist. Derselbe Name kehrt wieder in Cameria in Latium, Camerinum in Umbrien und der gens der Camertes. Dass diese Namen zu Kauåa gehören, wird auch dadurch bestätigt, dass sie im Griechischen mittleres a

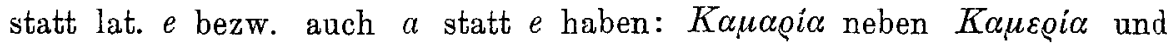

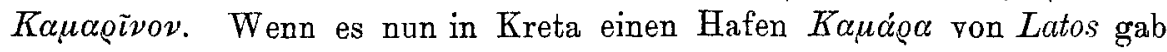

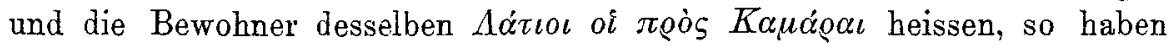
wir darin einen unverkennbaren Fingerzeig für den Ursprung von $L a-$ tium und Camars, Cameria ete. Dass beide Namen sich in engster Verbindung in Etrurien und Latium wiederholen, kann doch kaum mehr als Zufall angesehen werden, lässt vielmehr nur d i e e in e Deutung zu, dass

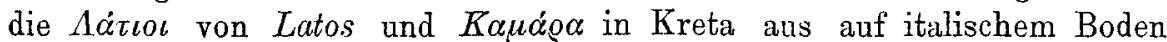

1) Für die Endung atos zieht Fick auch $\xi \xi$ 'O Orcius, Orcinius, Orchivius etc. Schulze S. 68 und 364 . 
kolonisiert haben. Die Latini sind also Söhne oder Verehrer der Lato; denn der insehriftliche Genetiv des Hafens Latos heisst $\Lambda \alpha \tau \tilde{\omega}$, der Dativ

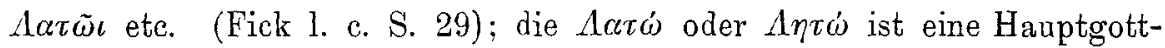
heit der älteren Bevölkerung Griechenlands gewesen, sie besass die ältesten Orakelstätten. Zu der nämlichen Sippe gehört ferner Camere ager in Bruttium (Ovid, Fast. III, 582). Auf Sizilien haben wir Kauáoıva,

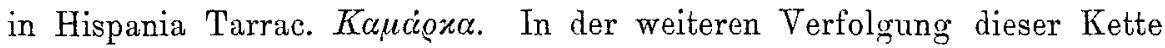
treffon wir die Kauc@ĩ $\iota \iota$ in Albanien zwischen dem Kaspischen und dem Schwarzen Meere, sie heissen genau ebenso wie die Bewohner von $\mathrm{Ka}$ -

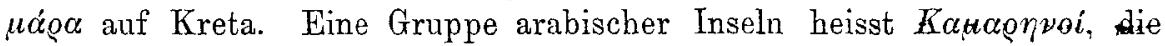
man als "Mondinseln" gedentet hat; denn kamar heisst arabisch "der Mond"; auch in Indien heisst eine Stadt Káúa@ an der Mündung des

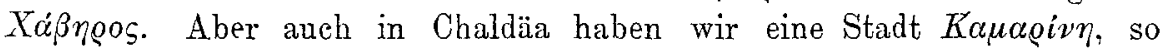
nennt Eupolemos Abrahams Vaterstadt $U r$ bei Euseb. prcep. ev. 9, 17 (Hommel, Grundr. S. 383). Diese Bezeichnnng der Stadt Ur rührt offenbar daher, dass sie eine Hauptstätte des Mondkults war. Es ist nicht unmöglich, dass auch den kretisch-etruskischen Namen desselben Stammes ein alter Mondgott zu Grunde liegt.

Kehren wir noch einmal nach Italien zurück, so batte Nissen aus der Uebereinstimmung des Namens Camars von Clusium mit demjenigen der Camertes ete. den Schluss gezogen, dass jener umbrischen Ursprungs sei ; dass das Verhältnis gerade umgekehrt liegt, erscheint mir wahrscheinlicher auf Grund des ganzen bei Schulze S. 139 ff. dargebotenen Materials, wenn auch nicht zu leugnen ist, dass auch umbrische und lateinische Ansprüche an diese Gruppe vorhanden sind.

Eine weitere Bestätigung für die Herkunft des Latinernamens aus Latos geben die Inschriften dieser Stadt, die verhältnismässig viel Namen bieten, die in Etrurien Verwandte haben:

Kó@ Vv Smlg. 5079 ist oben (S. 30) schon erwähnt. Die hexametrische Inschrift Smlg. 5083 rührt von einem Asंii $\omega \nu$ her, vgl. etr. auta CIE 369 , au Inal 384 (Arretium), autni 4304 (Perusia), antu $4250 \mathrm{ff}$., autles 1276 (Clusium), lat. Autius, Auvins, Autonins etc.

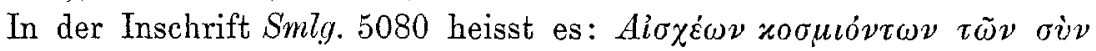

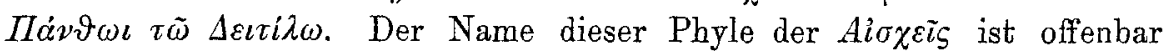
in etruskisch eszuma, Aescionius, Escionia, ferner vielleicht in Aeschiones, fundus Aeschinianus in Veleia und Rom, Esquilius vertreten; der Name

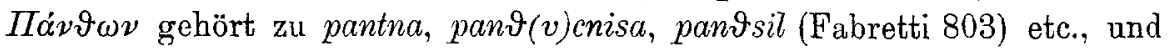

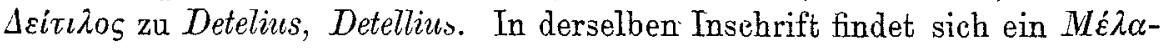
vos, möglich dass er zu griechisch $\mu \dot{\varepsilon} \lambda \alpha \varsigma$ gehört, wahrseheinlicher zu etr. melneal, meluta.

Und der $\Lambda v \sigma \sigma o \mu \varepsilon \dot{\nu} \eta \varsigma$ derselben Inschrift wird wieder einen etr. Namen mit $m n$ enthalten und zu der in Etr. zahlreich vertretenen Gruppe der Luseni, Lusius, Lussius etc. (Schulze 184), vielleicht auch zu lusce, luscni, 


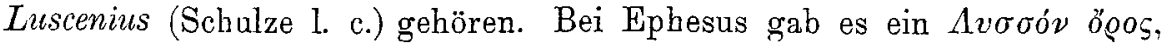

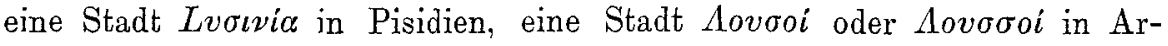

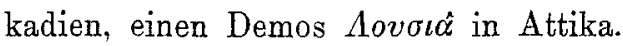

Smlg. Nr. 5075 enthält auch manches Vorgriechische, einiges wird im An-

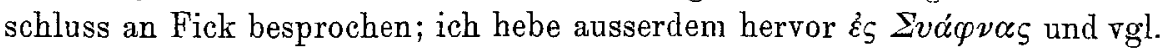
damit Suavithus, Suavettius, Suavitius, Suavitti mit dem etr. Suffix iఫi(e).

Fick S. 30: Der Fluss 'E $E \lambda \dot{\eta} \nu$ wird wohl ursprünglich ein $f$ gehabt haben, wie die Helena etruskisch auch Velena genannt wurde. Dann ist die bei Schulze S. 99 zusammengestellte Gruppe "Velina" heranzuziehen, die in ihren überaus zablreichen Mitgliedern auch Vellenus, Velenius, Vellenius, Vellinius zählt und schliesslich ebenso wie "Eגvoos Velurius zum Vornamen vel gehört.

Fiek S. 31: Zn $\Lambda a ́ \mu \omega \nu$ stelle ich nur folgendes gegenüber:

$\Lambda \dot{\alpha} \mu \omega \nu$ St. Kreta Ax́uos Ort und Fluss Kilikien Aáuvoos P. N. Latos Smlg. 5076 Ax́uv@os Fl. Lykien und Böotien

ad Lamnas b. Tibur

Lametus Fl. Bruttium

Lamyrus, Rutuler bei Vergil

Lamyrus und Lamyrianus, Etrusker Schulze S. 87

Á́uv@o Stadt Lykien

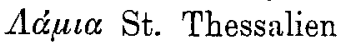

Aauias Athener

Ax́luos König der Lästrygonen

Aauivıov St. Hisp. Tarr.

Lamia Cognomen der gens Aelia

Á́uos, Rutuler bei Vergil.

Sovגia stimmt zu Sulenius, Sullenius, etr. zulus und sull(u)nica (Clusium), findus Solonianus und Solianus (Veleia). Eine Solonia Sabina ist Gattin eines haruspex Tuccius, „der Beruf ihres Mannes legt den Gedanken an etruskische Herkunft nahe" (Schulze S. 239). Sollonius begegnet in Verona und Mailand, Sollo CIE XI $670{ }_{683}$, Solonius und Sollius öfter.

" $A \lambda \alpha \sigma \sigma \alpha$ hatte ursprünglich wahrscheinlich ein $\mathcal{F}$ und ist abgeleitet von etr. vala, Vala und hat selbst Weiterbildungen in Valasenius, Valasinius, Valasennus (Schulze S. 376). Ohne Digamma reiht es sich einer andern, wie es scheint, auch etruskischen Gruppe ein, die bei Schulze S. 345 verzeichnet ist und $u$. a. einen Alasinius aufweist.

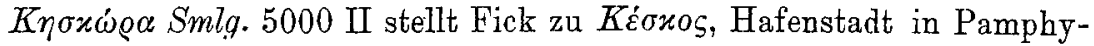
lien. Dazu wird die bei Schulze S. 353 gegebene Gruppe gehören: Casca, Cascius, Cascellius, Cascus, und was besonders zu beachten ist, das Cognomen Casco und Casconia. "Dass die Römer Casca zu cascus gedeutet haben, sagt Schulze l. c., ist selbstrerständlich, ob sie richtig gedeutet haben, ist eine andere Frage."

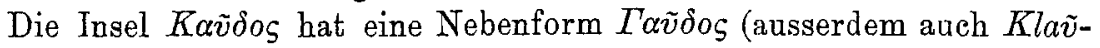

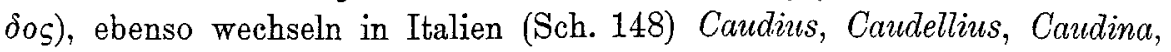
Cauden- mit Gaudienus, Gaudellius und weiter mit Cautius, Cautinus, Gautius, cantias, canvial, Cauthia. Diese Gruppe ist wieder ein schlagender Beweis für die Unfäbigkeit der Etrusker bezw. Kreter die Lautstufen 
der Mutae zu unterscheiden. Zur gens Caudia gehört das berïhmte Caudium im Hirpinerland (s. Schulze 560).

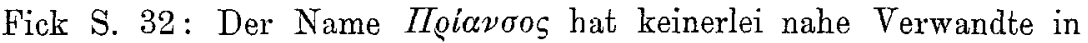
Etrurien, wohl aber gibt es eine etr. Göttin Pri, die besonders in der Inschrift von S. Maria di Capua mehrfach vorkommt, vielleicht leiten sich Privernum und Prifernum von ihr ab, denn die Namen auf fer sind etruskisches Sprachgut, cf. Caefer (Schulze 297), Meferna und Triferna (Schulze 107 n. 1 u. 378), Volfernu, velfercus, velfrei (Schulze 103 f.). Auch pricesa (Schulze 134), Briatia CIL XI 1865 u. a. mögen zu dieser Gottheit in Beziehung stehen.

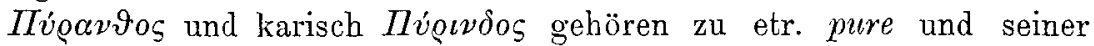
zahlreichen Familie (Schulze S. 217). In den kretischen Inschriften finden

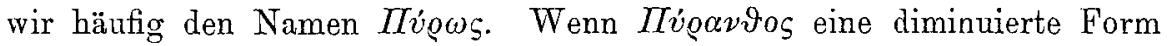
ist, entspricht ihr Purellius CIL 2368; die Inschrift ist aus Allifae, wo auch sonst etruskisch-lateinische Inschriften mehrfach vorkommen. Nahe bei Orvieto liegt ( $\mathrm{m} 0$ ) Porano (Schulze l. c.).

Für "Ivaros ist die Grundform Fivaros, Hesych nennt den Ort Eiva$\tau o \nu$, ebenso kennt er ein Eivarov in Lykien. Es ist klar, dass fivaros der Personenname ist, der dem Ort den Namen gab, wir treffen ihn wieder in Vinatius CIL XI $6712_{484}$; er gehört zu der weit verzweigten. Sippe, deren Stammvater vina hiess (Schulze 380).

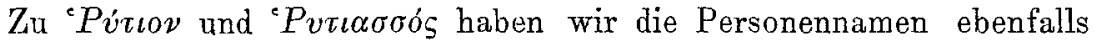
in Etrurien, und zwar in rutia CIE 2697 sqq. (Clusium), latinisiert Rutius CIL V 932, ferner rutsnei CIE 4083 ff. (Perusia) u. a. (s. Schulze S. 222).

"Die Nachbarstadt von Fivatos gegen Osten nennt Stephanos Bicv-

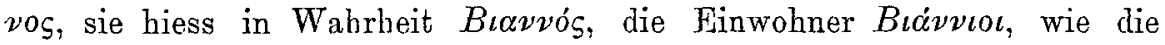
Inschriften zeigen, womit der heutige Name Viano ïbereinstimmt". Hiernach erklärt Fick es für fraglich, ob der Anlaut ursprünglich $B$ oder $F$ ist. In Italien haben wir entsprechende Formen mit $b$ und $e$, und zwar Bienus, Biennus CIL XI 972 (Regium Lepidum), Bienus VI 10006, 13584, Biennius CIL VI 13584. Scluulze S. 133 hat mit diesen Namen auch Benus verbunden, das wir oben (S. 32) zu $B \dot{\eta} \nu \eta$ oder $B \dot{v} v v \alpha$ gezogen haben. Die Namen mit und ohne $i$ sind mit Rücksicht auf die beiden kretischen Orte wohl zu sondern. Biaina, den inschriftlichen Namen des Chaldenlandes am

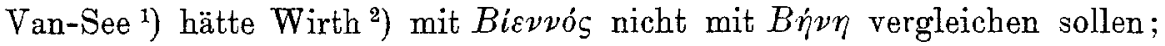
in der Tat ist ein Zusammenhang zwischen beiden nicht unwahrscheinlich, da nach Lehmann-Haupt ${ }^{3}$ ) die Biainier aus dem Westen Kleinasiens nach Armenien gewandert sein sollen.

1) [S. dazu besonders die grosse Inschrift König Rusas' II Z. 41, und die Bemerkungen dazu $Z D M G 56$ (1902), S. 114, vgl. Bd. 58 (1904), S. 831 . C. F. L.-H.]

2) Politisch-Anthrop. Revue, l. c. S. 233.

3) Materialien zur älteren Geschichte Armeniens, Göttingen 1907, S. $120 \mathrm{ff}$. und Sitzungsber. der Berliner archäol. Ges. Nov. 1907 Nr. 32, S. 56 ff. 


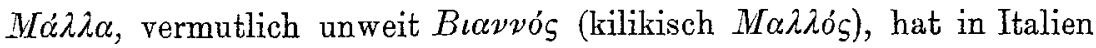
eine grosse Sippe maalnaś CIE 4083, malamenaś C1E 177 (Sena), malavisina CIE 2570 (Clusium) sind etruskische, Mallius, Malanius, Malinius, Maletini, Malisini, Malonii latinisierte Formen, vielleicht gehört auch Malo CIL 3924 dazu (s. Schulze S. 313).

Fick S. 33: Kv́ $@ \beta \alpha$ (auch in Karien und Pamphylien) alter Name für

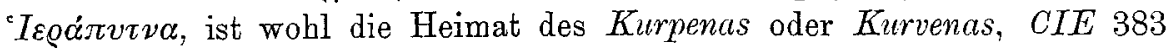
(Aretium), der Curpenii und der Curpennii. Wahrscheinlich ist Kúgßa direkt identisch mit curve CIE 1797, 2060 sq. (Clusium), und es gehören dazu auch die Curvinii und Curvii. Die Formen curspena CIE 1458 (Clusium) und curspic 4311 (Perusia) gehören nach Schulzes Vermutung vielleicht auch hierher, äbnliche Beispiele eines eingeschobenen $s$, dessen Funktion uns einstweilen dunkel bleibt, hat Schulze S. 156 mehrere angeführt. $K \dot{v} \varrho \beta \alpha$ lebt weiter im modernen Ortsnamen Corbignano bei Florenz.

In dem Hauptorte am Ostende Kretas, ${ }^{\text {wo }}$ sich die Urkreter am

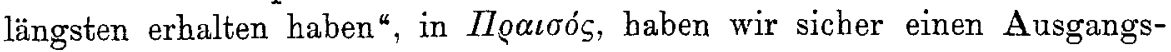
punkt für zahlreiche etruskische Gesehlechter. In Clusium baben wir die einfachste Form presu, dessen $e$ wie häufig im Etruskischen über $e i$ aus ai entstanden ist, was durch die Formen preisnte (neben presnte) und die lateinischen Formen Praesidius (neben Presidius), Praesentes und das gerade auf etr. Gebiet sehr oft vorkommende Praesentius erwiesen wird. Die Form preisnףe, die bei Schulze S. 210 Anm. 6, wo die übrigen zu finden sind, noch fehlt, steht jetzt CIE 5102 (Orvieto).

Aus dem Vertrage zwischen Itanos und Praisos seien erwähnt: $\xi \varsigma$

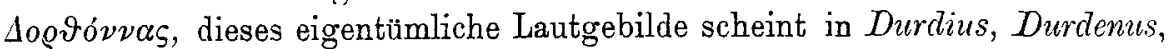
Durdenius, Turtellinus, Turturius, etr. turte (CIE 3007) und turtia 2975

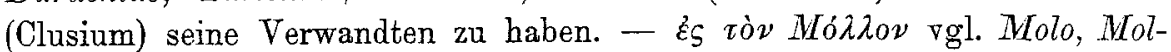

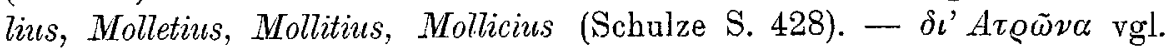
atru, atrunias, Atronius (Schulze S. 269).

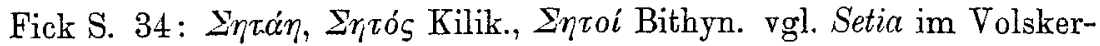

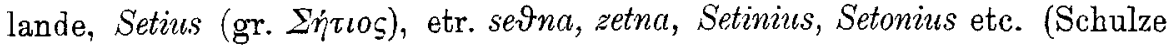
S. 231 und 560$)$.

"Aбos. Dazu gehört wohl nicht etr. asi, da es nach Schulze S. 214 aus axsi entstanden ist, wohl aber asna, asati, osk. Asis, Asinins etc. (Schulze 129).

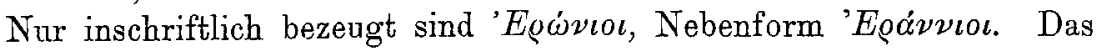
letztere veranlasste mich, den Namen im heutigen Veran Episcopi zu suchen, das seinen Beinamen vielleicht führt zur Unterseheidung von dem nicht gar weit entfernten Veriana. Dann hat der Name ein Digamma, und es scheint höchst wahrscheinlich, dass er mit Verona, das eine Etruskerstadt war, identisch ist, obwohl das $e$ in Verona lang ist. Dieses ist "direkt identisch mit etr. veru" (Sch. S. 574), das zu einer sehr verbreiteten Sippe gehört, vgl. Feromius CIL XI 3943 (Capena), X 4890 (Vena- 
frum), auch Verronius IX 362, 407 (Canusium). Doch kommt auch Eronius CIL XI, 6045 vor.

Kavvos, St. in Kreta und in Karien, wohl entstanden aus Ka.Fvos, gehört zu Caunius, Gaunia, cavinei (Volaterrae), Cavinius, Gavinius etc. (Sehulze S. 76). Der Name cavinei wird später zu cuinei, wie schon oben erwähnt; dies bringt uns auch Auflklärung über den Namen des Lapithen-

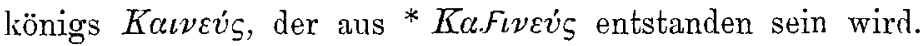

'O $20 \tilde{v}_{\varsigma}$ hält Fick S. 13 für griechisch und bringt es zu fólos, das wohl "Rundstein" heisse, in Beziehung; er nimmt zwei ursprïngliche Digamma

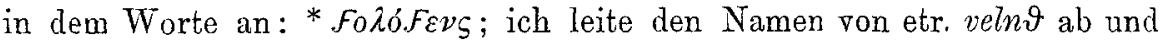
glaube unter Berufung auf die Wiedergabe dieses Namens in pränestinisch Volntilius, Volentilius und Voluntilius, dass sich hierauf sämtliche überlieferte griechische Formen des Namens der Stadt und ihrer Bewohner zurückführen lassen. Wir besitzen aus Olus ein paar Inschriften, die mehrere vorgriechische Namen enthalten. So finden wir Smlg. 5107 einen Tvqáoıos, der einen Namensvetter aus Mallos in Smlg. 4941 (Ap-

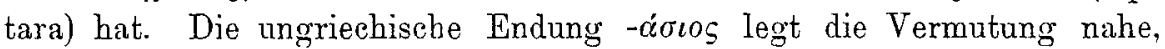
dass der Name nur gräzisiert ist und mit Tízך nichts zu tun hat; dann gehört er zur Gruppe Ducenius (Schulze S. 160), zu etr. tucmenas, und es

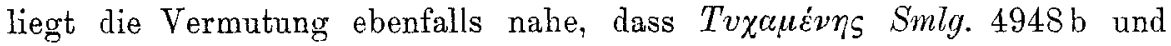

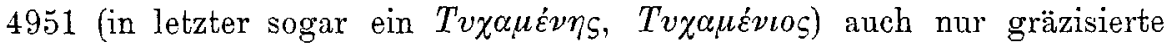
Formen eines ungriechischen Namens mit - $\mu \nu$ a sind, so dass sogar die direkte genaue Entsprechung dafür in tucmenas vorliegt, wofür wieder das oben zitierte tucumsna nur eine Variante ist. Eine andere Inschrift aus Olus (Smlg. 5104) lehrt uns einen Daniurgen 'Aorias kennen, vgl. silva Arsia, Arsenius, Arsellius, Arsnius, Arsius, Arsina, arzni, Arsaniacus. Genau so wie der Damiurg aus Olus heisst griechiseh der Fluss Arsia, der Grenzfluss von Oberitalien und Illyrien. 'Aogía heisst aueh die Umgegend vom See "Alo $\sigma \sigma a c$ (Van-See), wo die hattidischen Biainier oder

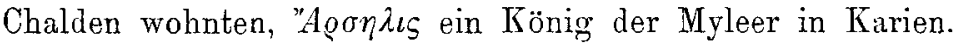

Dieselbe Inschrift Smlg. 5107 macht uns auch mit einem Damiurgen AoxQivos bekannt. Stammt der aus Lokris? Vielleicht. Aber die Lokrer waren nach der Ansicht der Alten Leleger, und derselbe Name kann sich auch anderswo bei stammverwandter Bevölkerung entwickelt haben. $\mathrm{Zu}$ der lelegischen Namengruppe, zu der die Loxgoi ge-

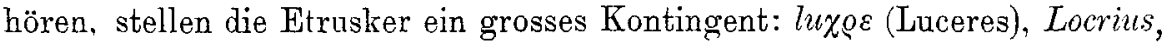
Lucernius Lucretius (gr. Aoxoýrıos), Locer(iuss?), Lucrianus, Lucrinius, fundus Locresianus in Veleia.

Ein Grabstein aus Olus, Smlg. 5108, trägt die Inschrift: $\Phi i \lambda_{l \varsigma} K \eta$ -

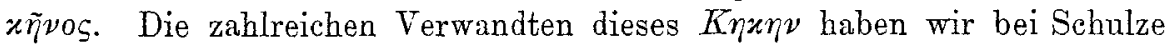
S. 273: Cecanias CIL XI $6712_{108}$, Geganius 6223, Gigennius (das Schulze S. 220 einen "ganz etr. Namen" nennt) CIL VI 2379a, zahlreich in Sas- 
sina, ferner vielfach etr. cicu und cicunia, Gigennaus CIL VIII s. 18065, das Quartier der Cicinenses in Rom etc.

Eine Inschrift von Elyros Smlg. 4960 macht uns mit einem Kosmen $T u ́ \varrho \beta a \sigma o s$ bekannt; der Name hat besonderes Interesse; da er nicht griechisch ist, so ist auch das Fest Túgß $\eta$, das auf dem Chaongebirge zwischen Argos und Tegea dem Dionysos gefeiert wurde (Paus. II, 24. 6), nicht

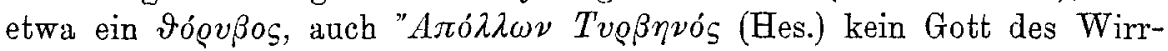
warrs, sondern wir haben in diesem Namen einen alten Gott zu suchen, an dessen Stelle in Argolis der Dionysos getreten und der anderswo mit

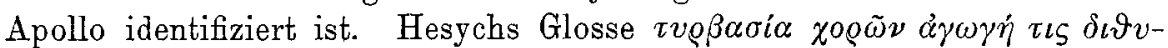

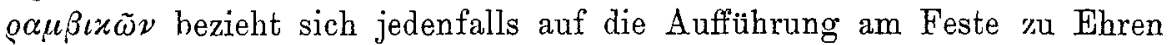

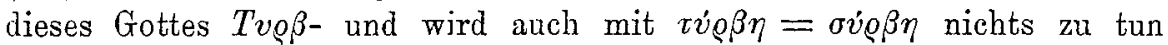
haben. Ein Verehrer oder Nachkomme dieses Gottes steckt im Namen

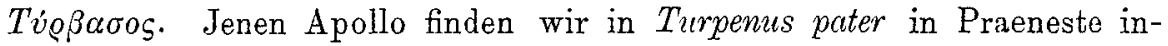
schriftlich wieder, also in einer etruskischen oder halbetruskischen Stadt; seine Nachkommen oder Verehrer finden wir an vielen Orten als Turpiliz, einen Turpilinus in Falerii, einen Turpilienus CIL VI 27774, etr. turpli in Perusia, auch je zwei Turpidii und Turpedii und zweimal das Cognomen Turpio (bei einem Ambivius und einem Antistius), s. Schulze S. 246 und 308. In Kreta begegnet von demselben Stamme noch ein Tv@ßaĩos in der Inschrift aus Hyrtakina 5055 a.

Zwischen Kreta und dem Peloponnes lag eine kleine Insel namens

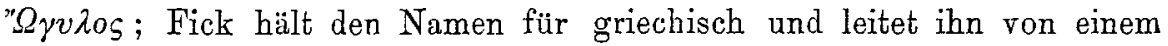
Adjektiv $\dot{\omega} \gamma \dot{\gamma} \lambda \mathrm{S}_{\mathrm{S}}=\mathrm{krumm}$, gebogen ab, das in $\dot{\omega} \gamma \dot{v} \lambda \lambda o v \tau o=\sigma v \nu \varepsilon x \alpha \dot{\mu} \mu \tau-$

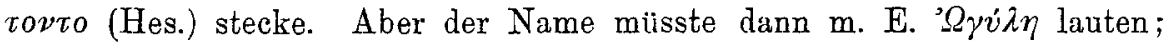
der Name wird vielmehr vorgriechisch sein; dann haben wir in dieser Insel wahrseheinlich die Heimat der plebeischen gens Ogulnia, wie aus folgenden Inschriften klar erhellt (Schulze 150 f.): Oculnius CIL 2097 (Clusium), 4901 (Spoletium) VI 23427, ferner Oculnius auf Münzen, Ogulnius CIL 6064 (Minturnae), XIV 1423 Ostia, 3979 f. Nomentum, L. Ogulni V $8112_{62}$, Oglinia CIE $2075=$ CIL XI 2479 (Clusium), uclina CIE 4502 (Perusia), uclnial 2571 (Clusium), Oc(u)latius (c. $12 \mathrm{mal}$ ) und Oclctinius cons. 218. Ob die übrigen Formen, die Schulze S. 150 n. 4 heranzieht, a(u)cilnia, auclina, acline, und der oskische Vorname Aukil nebst Ocellius, Aucilius, sowie Aucideni and Auceius auch hierher gehören, ist leider nicht zu entscheiden. Die Formen mit au führten Schulze zu der Vermutung, dass es möglicherweise zwei verschiedene Ogulnii gegeben habe, einen mit kurzem und einen mit langem $o$. Da wir das Analogon von $R \alpha \tilde{v} \varkappa o \varsigma, R \tilde{\alpha}-$ nos, Rüxos haben sowie von rafi, raufi, rufi, so können sämtliche obige Formen mit $l$ auf das dem griechischen " $\Omega \gamma v \lambda o s$ entsprechende vorgriechische Wort zurückgehen, die Quantität dürfte bei der Uebertragung des fremden Wortes ins Lateinische kaum ins Gewicht fallen. Wenn aber Ogulñius zu Aucidius gehört, dann kann eine erst auf italischem Boden vorge- 
gangene Namenbildung aus dem Stamme Auc- unter anderem auch zu dem Namen Oculnius geführt haben, so dass wir in der Insel " $\Omega \gamma v \lambda o \varsigma$ nicht die Heimat aller jener Etrusker zu sehen hätten, sondern nur dieselbe Namenentwicklung. Allerdings wäre ja auch eine Rückkehr zu einfacherer Namensform wieder denkbar, so dass alle Träger obiger Namen doch aus

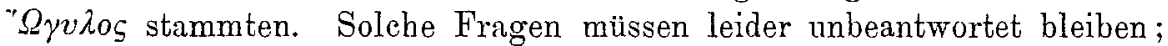
sicher ist, dass die Ogulnier, wie schon Schulze ausdrücklich feststellte, etruskisch und, wie ich nunmehr hinzufügen darf, hattidisch sind.

Das Ergebnis unserer Untersuchung lässt sich in wenige Worte zusammenfassen:

Die altkretischen Ortsnamen scheinen durehweg mit Personennamen in Zusammenhang zu steben. Die grosse Massedieser Ortsnamen. insbesondere die $\mathrm{Na-}$ men fastaller (vielleichtaller!) an der See liegenden OrteKretaskehren in italischen Orts- und Personennamen wieder, die etruskischen Ursprungs sind, ebenso ein grosser Teil gelegentlich vorkommender kretischer Personennamen. Es muss daher eine ausserordentlich starke Einwanderungin Italien aus Kreta oderaus Ländern erfolgt sein, die eine mit der altkretischen stammverwandte Bevölkerung hatten. Es bedarfnurnoch einer genaueren lieststellung des Anteils, den die übrigen Länder und Inselndes ägäischen Meeresander Besiedelung Italiens gehabt haben.

Gelsenkirchen. 